\title{
A unique presentation of fahr syndrome secondary to chronic hypoparathyroidism: A case report
}

Idiopathic basal ganglia calcification, or Fahr Syndrome, often presents in middle-aged patients presenting with parkinsonian symptoms. Unique presentation of young patient presenting with a seizure, and without the presence of parkinsonian symptoms. Imaging revealed near complete calcification of bilateral basal ganglia. Most common etiology of basal ganglia calcification is idiopathic or familial, but may also be secondary to metabolic derangements.

KEYWORDS: fahr syndrome, idiopathic basal ganglia calcification, cerebral calcification.

\section{Introduction}

Idiopathic Basal Ganglia Calcification (IBGC), also known as Fahr syndrome or bilateral striatopallidodentate calcinosis, is an uncommon neurodegenerative disorder defined as the accumulation of calcium deposits throughout the basal ganglia structures, as well as other central nervous system regions. Both familial and non-familial cases have been reported, with an autosomal dominant inheritance pattern [1]. Idiopathic hypoparathyroidism has also been implicated in the majority of cases, however the pathogenesis of deposition of calcium in the basal ganglia remains unknown [2]. The mean age of symptom onset is $40 \mathrm{y}$ with men presenting with double the prevalence rate as women [3]. The most common presenting signs and symptoms of the disorder are cognitive impairment, psychiatric symptoms, and motor symptoms, all of which are present in 50-60\% of symptomatic patients $[1,3]$. Isolated seizures are uncommon, and has previously been reported in a case report of an infant, found to have idiopathic hypo-parathyroidism [2].

\section{- Case report}

A 25 y female with no past medical history presented to the trauma department following a witnessed seizure and fall. She had two prior seizures within two months and a possible seizure with minimal associated workup at age 11 , which revealed no abnormalities. The patient had not underwent any brain imaging at that time. She has never taken seizure medications and has no family history of seizures or Fehr syndrome. Neurological exam reveals mildly increased tone on the left, but otherwise no abnormal findings. Patient had not previously complained of parkinsonism, chorea, dystonia, or other motor symptoms.

\section{Imaging and diagnosis}

Computed tomography revealed bilaterallysymmetric calcifications involving the entire basal ganglia, cerebellar dentate nuclei, anterolateral superior and thalamic pulvinars, and the lateral subcortical white matter of the centrum semi-ovale (FIGURE 1). The presence of bilateral diffuse basal ganglia calcification on brain imaging is necessary and sufficient for the diagnosis of idiopathic basal ganglia calcification.

\section{Hospital course and follow up}

Admission laboratory studies were notable for a calcium of $4.3 \mathrm{mg} / \mathrm{dl}$, phosphorus of $5.5 \mathrm{mg} / \mathrm{dl}$ and parathyroid hormone level of $6 \mathrm{pg} / \mathrm{ml}$. She was started on levetiracetam for seizure control, laboratory abnormalities were corrected, and endocrine consulted. Imaging and laboratory studies are suggestive for chronic hypo-parathyroidism as the cause of secondary Fahr syndrome. The patient was followed by endocrinology, and was put on calcitriol and calcium carbonate. Her hypo-parathyroidism was thought to be of autoimmune etiology, as there was no history of thyroid surgery, or other syndromic findings.

\section{Discussion \\ Clinical presentation, genetics and pathophysiology}

IBGC is often found in autosomal dominant

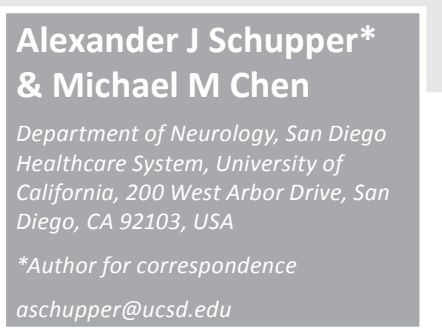




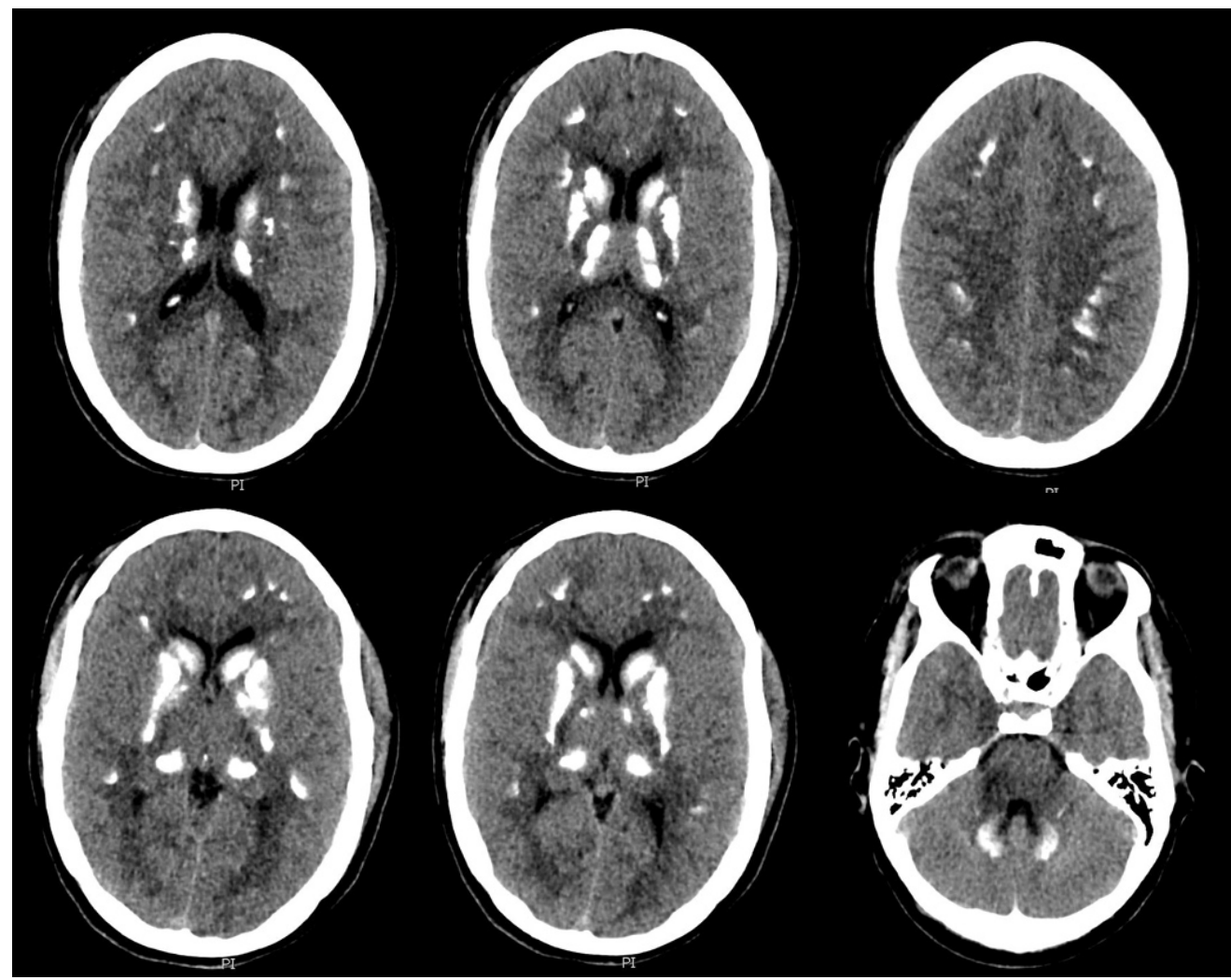

Figure 1. Axial CT images demonstrating dense, bilaterally-symmetric calcifications involving the entire basal ganglia, dentate nuclei in cerebellum, anterolateral superior thalami, thalamic pulvinars and the lateral subcortical white matter of the centra semi-ovale.

families, but has also been reported in sporadic cases. Most patients are diagnosed in middle age, and men are twice as likely to be diagnosed as women [3]. Here we present a young woman with no affected family members presenting with diffuse basal ganglia calcifications. Calcifications most commonly reported in the globus pallidus, but may be present in the centrum semi-ovale and cortical white matter, as seen in our patient. The presence of extrabasal ganglia calcification may be the cause of this patient's unique presentation of multiple seizures. The majority of patients present with symptoms of parkinsonism and movement disorders, cognitive decline, and psychiatric symptoms $[1,3,4]$.

IBGC has a strong genetic link, with a familial autosomal dominant inheritance being found most commonly. SLC20A2 on chromosome 8p11.2 and PDGFRB on chromosome 5q32 have been the most commonly implicated genetic mutations, though other genes have led to similar phenotypes [1].

Basal ganglia calcification is most commonly thought to be due to endocrine disorders, specifically disturbances in the regulation of parathyroid hormone. Hypo-parathyroidism can lead to increased calcinosis, and it is believed that calcium deposition begins in the vessel wall, eventually extending to the neuron. This deposition of the basal ganglia leads to impaired local circulation, causing additional neuronal injury and subsequent increased calcium deposition [5].

\section{- Differential diagnosis}

In determining the diagnosis of IBGC, one must also consider the various causes of intracranial calcifications. Often the clinical presentation and location of calcification on brain imaging may be helpful in determining the diagnosis. Basal ganglia calcification must be present for the IBGC diagnosis, and the globus pallidus is almost universally affected. Patients most common present with either cognitive, psychiatric or parkinsonian symptoms, however, patients may present with seizures (as seen in our patient), headache, stroke, vertigo, paresis, or other neurological presentations. 
To diagnose IBGC, other causes must be ruled out. The many etiologies of intracranial calcifications, including infectious, metabolic, congenital and vascular, must be further explored in determining the cause of basal ganglia calcifications. For example, the TORCH infections (toxoplasmosis, other, rubella, cytomegalovirus, herpes simplex virus) must be considered, as they may cause intracranial calcifications. Most commonly, metabolic derangements causing problems with calcium homeostasis are a common cause of basal ganglia calcification. Serum levels of alkaline phosphatase, calcium, calcitonin, parathyroid hormone, as well as infectious labs and heavy metal concentrations are recommended.

\section{- Management}

Currently there is no treatment for IBGC, and management is limited to supportive care for presenting neurological and psychiatric symptoms. If underlying metabolic disturbances are found, as seen in this patient, correctly underlying abnormalities may improve the presenting symptoms. Chelators with antioxidants and calcium antagonists have been reported, however, these results have not been confirmed [6]. For the rare patient presenting with seizure activity, antiepileptic drug use with medications such as levetiracetam are appropriate. In a young female patient of childbearing age, it is important to limit the use of teratogenic medications including valproic acid and carbamazepine.

\section{Conclusion}

Fahr syndrome is a rare neurodegenerative disease defined by the presence of bilateral basal ganglia calcifications. Most cases are familial, and there is a strong genetic component to the disease, specifically an autosomal dominant inheritance. Patients commonly present with cognitive, motor and/or psychiatric symptoms, but may present in a variety of phenotypes. Idiopathic basal ganglia calcification may present similarly to other infectious, metabolic, congenital or vascular disorders affecting the nervous system, and an exhaustive workup must be conducted to rule out other causes.

\section{REFERENCES}

1. Nicolas G, Pottier C, Charbonnier C et al. Phenotypic spectrum of probable and genetically-confirmed idiopathic basal ganglia calcification. Brain. 136, 3395-3407 (2013).

2. Bhat MA, Laway BA, Mustafa F. Bilateral basal ganglia calcification and recurrent generalized seizures as initial presentation of idiopathic hypoparathyroidism in an infant.

\section{J. Pediatr. Neurosci. 10, 178-180 (2015).}

3. Manyam BV, Walters AS, Narla KR. Bilateral striopallidodentate calcinosis: clinical characteristics of patients seen in a registry. Mov. Disord. 16, 258 (2001).

4. Mufaddel AA, Al-Hassani GA. Familial idiopathic basal ganglia calcification (Fahr's disease). Neurosciences. 19, 171-177 (2014).
5. Saleem S, Aslam HM, Anwar M et al. Fahr's syndrome: literature review of current evidence. Orphanet. J. Rare. Dis. 8, 156 (2013).

6. Skvortsov IA, Rudenskaia GE, Karaseva AN et al. Effectiveness of therapeutic use of complexones in various diseases of the extrapyramidal system in children. Zh. Nevropatol. Psikhiatr. Im. S. S. Korsakova. 87, 1457-1462 (1987). 